\author{
Andrew C. Singer ${ }^{1}$, David E. Crowley ${ }^{2}$ and lan P. Thompson ${ }^{1}$ \\ ${ }^{1}$ Centre for Ecology and Hydrology, Oxford, Mansfield Rd, Oxford, UK, OX1 3SR. \\ ${ }^{2}$ Dept of Environmental Science, University of California, Riverside, California, 92521, USA.
}

For millennia, secondary plant metabolites have antagonized microorganisms, insects and humans alike, ultimately generating a complex and dynamic mixture of facultative and obligate interactions from symbioses to pathogenicity. Secondary plant metabolites have an important role in developing the myriad of organic pollutant-degrading enzymes found in nature. The link between secondary plant metabolites and enzymatic diversity has yet to be exploited, with potential applications in fields as varied as pest management, bioremediation and fine chemical production.

Between 1997 and 2002, >600 research papers supported the observation that the addition of a plant to a contaminated medium (e.g. soil, wetland and hydroponics) enhances the removal and/or transformation of a pollutant. However, the mechanisms are often left unresolved. The paradox is that most of the persistent pollutants that are bio- and phyto-remediated are novel man-made chemicals (xenobiotics), which are thought to be different enough from natural compounds to preclude their expeditious biotransformation. Here, we present published evidence for why bioremediation and phytoremediation are proving successful. We provide a survey of relevant interdisciplinary research to place the latest developments in microbial-mediated biotransformation and remediation in perspective. We present our hypothesis in an evolutionary context, suggesting that the interactions between plants, insects and microorganisms have indirectly generated a diverse array of catabolic enzymes, many of which are perfectly suited to metabolising and detoxifying xenobiotic compounds. We discuss studies that investigate novel techniques of stimulating xenobiotic degradation in microbial populations and conclude with possible avenues for exploiting secondary plant metabolites (SPMEs) in biotechnology and remediation.

At the outset of the industrial revolution the amount of wood, coal and other fuels that were being burnt increased massively. The resulting pollutants, although natural products, were provided in vast excess to 'natural' rates of formation, which, in combination with their innate recalcitrance to degradation, meant that these compounds accumulated in the environment. The presence of decomposing organic matter, (unrefined) fossil fuels and volcanism continually produce a wide range of chemicals (e.g. polycyclic aromatic hydrocarbons; Fig. 1; 2,3) referred to as persistent organic pollutants when of anthropogenic origin. Common pyrolytic and polymerization reactions further extend the range of 'natural' pollutant analogues [1]. Although we recognize the potential importance of such affecters, we will not focus on them here. Rather, we present less investigated mechanisms that, on further study, might prove significant in exploiting the xenobiotic biodegradative capacity of microorganisms.

\section{Plant root exudates}

All plants modify the surrounding soil (rhizosphere) through the release of organic and inorganic substrates. Roots receive $30-60 \%$ of the net photosynthetic carbon, from which $10-20 \%$ is released by rhizodeposition [2,3]. Exudates consist primarily of low molecular weight (LMW) and high molecular weight (HMW) organic acids. Typically, the total concentration of organic acids in roots is $10-20 \mathrm{mM}$, largely consisting of lactate, acetate, oxalate, succinate, fumarate, malate, citrate, isocitrate and aconitate [4]. The remainder of organic solutes in roots is composed of sugars $(90 \mathrm{mM})$ and amino acids $(10-20 \mathrm{mM})$ [4]. The HMW organic acids are mucilage and ectoenzymes [5]. The exudates cause rhizosphere-inhabiting microbial populations to increase well beyond those of the bulk soil [4], attracting motile bacteria and fungal hyphae that stimulate an array of positive, neutral or negative interactions with plants [6]. Decomposition of organic acids and many other compounds is routinely found to be two to three times greater in the rhizosphere than in the bulk soil [4].

\section{Secondary plant metabolites}

Plants produce a diverse array of $>100000$ low molecular mass secondary metabolites; estimates of the total number in plants exceed 500000 [7]. By nature of being secondary metabolites, they are traditionally considered to be nonessential for the basic metabolic processes of the plant [8]. Among the most widespread of the volatile, non-floral secondary metabolites is isoprene [9], which is the building block for the more complex chemical analogues, terpenoids, of which there are currently $>15000$ [10]. Monoterpenoids (Fig. 2; 23-29), of which there are more than a thousand naturally occurring, are the simplest family of 


\section{Glossary}

ALLELOPATHY - The release of a chemical by a plant into the environment, which adversely impacts a competing plant.

AROCLOR 1242 - A commercial mixture of polychlorinated biphenyl originally manufactured by Monsanto Co., where the 12 represents the number of carbons, and the 42 is the average percentage chlorine per biphenyl (w/w). ATROPISOMER - An enantiomer of a reference compound that only very slowly converts to the reference compound at environmental temperatures.

COMETABOLISM - A metabolic process where an enzyme produced for the metabolism of a primary substrate (cometabolite) is capable of fortuitously degrading a secondary substrate affording no additional energy or nutritive gains from the secondary process. Common examples include biphenyl and PCBs, and methanol and trichloroethylene, where the former of the pair is the cometabolite and the later is the recalcitrant compound, which is cometabolised.

COMETABOLITE - A compound that provides the energy and nutritive substrate for a microorganism to carry out cometabolism.

ENANTIOMER - Two or more chemicals with the same formula and chemical bonding but have nonsuperimposable mirror images, analogous to human hands.

ENANTIOSELECTIVITY - The capacity attributed to something (e.g. enzymes) that differentially recognizes chemical enantiomers.

INDUCER - We use this term to refer to a chemical that stimulates the degradation of a recalcitrant pollutant by an unknown mechanism. An inducer can stimulate biodegradation by means of cometabolism, growth-linked metabolism, or through a more complex cascade of mechanisms within the host.

isoprenoids and are synthesized by the linkage of two isoprene units [11]. Terpenes have been explored for a range of uses including as anti-bacterial, anti-fungal and anti-cancer compounds $[8,12,13]$. The largest family of terpenoids are the sesquiterpenoids (Fig. 2; 33,42), of which more than one hundred skeleta are known, and several thousand of these compounds have been identified [13]. Sesquiterpenoids have a diverse range of biological activities including antifeedant, toxic and antibiotic substances, insect juvenile hormone mimics, phytoalexins (anti-fungal), plantgrowth regulators and antioxidants [8].

Members of the SPME family of phenolics comprise another major group of plant-derived compounds. Plant phenolics are biochemically formed via the shikimate pathway, which produces the group of phenolics called phenylpropanoids, of which hydroxy-cinnamic acids (Fig. 2; 32) and coumarins are members [14] (Fig. 2; 37,38). Coumarins are a very common secondary plant metabolite [15], of which $>800$ have been identified. They consist of substituted benzene rings commonly hydroxylated, alkylated, alkoxylated, or acylated and have a range of toxic properties that have, in some cases, been shown to be exacerbated by exposure to UV light [15]. Hydroxycinnamic acids occur in many different forms and when polymerized or dimerized can form lignin, lignan and neolignans [11]. The most common of all plant phenolics, however, are the flavonoids (Fig. $2 ; \mathbf{3 5 , 3 6}$ ), which form the skeletal backbone of the polyphenolic class of proanthrocyanidins (tannins).

\section{SPMEs as a defense against herbivory}

It has been suggested that many SPMEs evolved for functions including frost tolerance, ALLELOPATHY (see GLOSSARY), nutrient storage, structural reinforcement and signaling to mutualists [16]. There is also strong evidence that biotic variables can influence the chemical makeup of plants, such as interactions between plants and microorganisms or insects. Herbivory has most notably been shown to cause shifts in the production of ethylene, methyl salicylate and methyl jasmonate [9] (Fig. 2, (34)). Herbivore-induced chemical release is also commonly observed for many other secondary metabolites such as $\alpha$-tomatine [17], linalool (Fig. 2; 24), $\alpha$-pinene (Fig. 2; 28) and (E)- $\beta$-ocimene [18-22]. The literature suggests that, in addition to the floral volatiles that are designed to attract particular insects, a mixture of terpenoids and other compounds is emitted from the plant, enabling the predator to distinguish between infested and noninfested plants [19]. Plants can respond to herbivorous activity by producing volatile substances that attract parasitoids of the herbivore $[23,24]$. The induced release of volatile chemicals could be a mechanism of indirect plant defence, both as a biological control signal and as a deterrent of further herbivory $[19,24]$. Several reviews help towards understanding the debate regarding the link between volatile emissions and herbivore feeding [17,18,24-26].

Microbial associations, such as plant growth-promoting bacteria or plant pathogens, have also been shown to induce the production of phenolics in plants and their roots $[27,28]$. In addition to producing a battery of chemical defenses itself, the plant might also foster mycorrhizal and endophytic fungal associations resulting in improved growth, resistance to stress and herbivory [29,30]. Research suggests that herbivory stimulates the production of toxins by the endophyte, which deters, inhibits or kills the herbivore [31]. The endophyte thereby contributes to the already diverse array of chemical defenses herbivores must overcome.

\section{Microorganisms as a defense against secondary plant metabolites}

"The process of co-evolution between plants and their natural enemies - including viruses, fungi, bacteria, nematodes, insects and mammals - is believed by many biologists to have generated much of the Earth's biological diversity" [16]. One could argue that co-evolution dictated by the 'direct defence' approach taken by some plants (i.e. the production of primary and secondary metabolites) $[19,32,33]$ might have helped to foster the extremely complex and intimate relationship between insects and mutualistic bacteria. Such interactions date back to the origins of the host families or superfamilies (100-250 million years ago) [34]. In addition to a microbe providing the host insect with essential amino acids [35], a herbivore might find partial relief from the toxic effects of SPMEs if an associating microorganism can use or detoxify the offensive chemical(s). Thus, the insect minimises the exposure risk when feeding on otherwise chemically unattractive plants. It is the continued modification to SPMEs over several millennia, necessary because of resistance by the insect and/or the resistance provided by the endosymbiont, which drives the development of loosely specific detoxifying enzymes. Hence, we postulate that, in a world without herbivory, plants would release a less diverse array of SPMEs, thereby relieving the pressure on herbivores and microorganisms to continually detoxify novel substrates. The xenobiotic-degrading capacity of microorganisms in such a world might have precluded many of our recent industrial and intellectual 


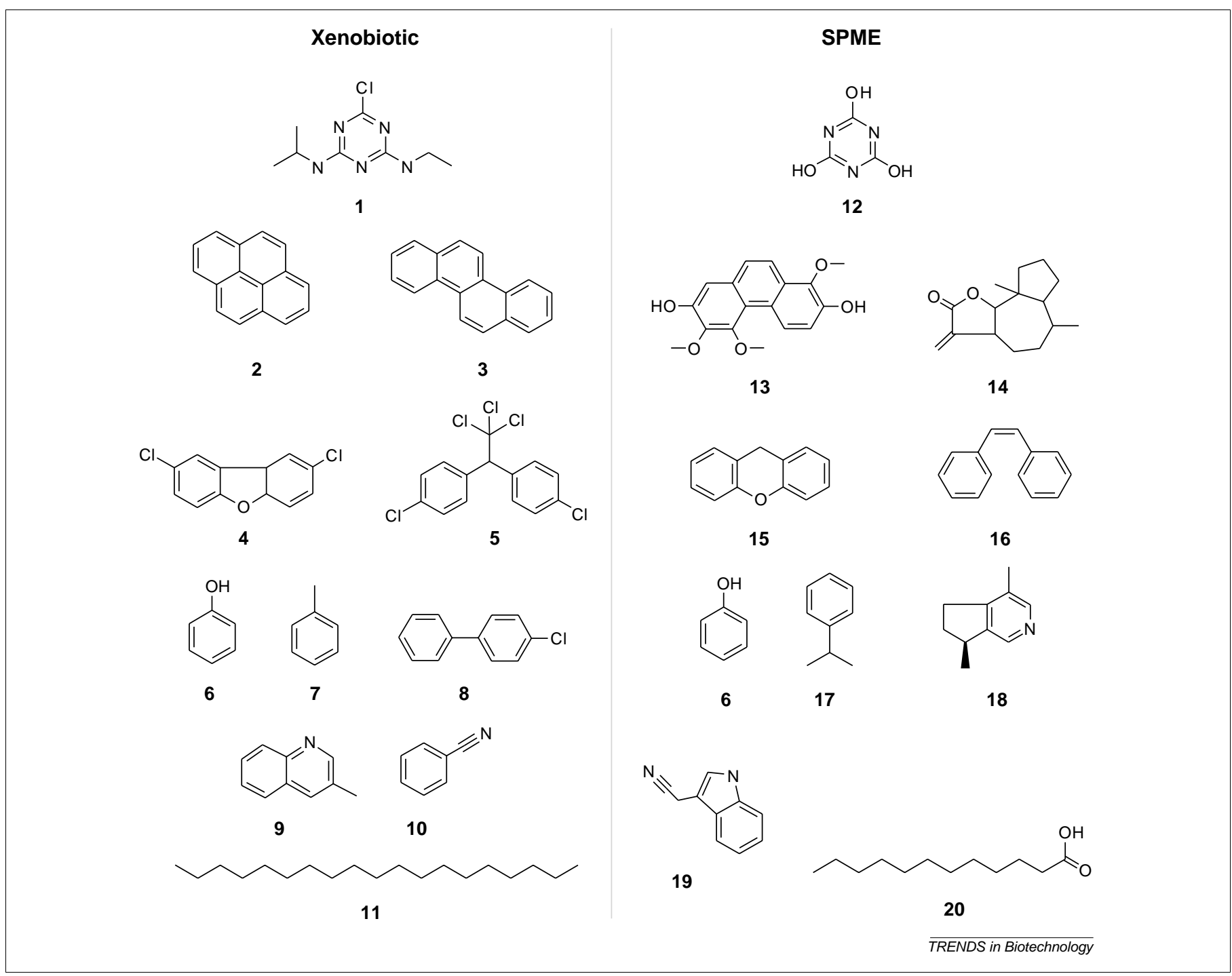

Fig. 1. Xenobiotics structurally analogous to secondary plant metabolites. (1) Atrazine; (2) Pyrene; (3) Chrysene; (4) 3,8-Dichlorodibenzo-p-dioxin; (5) p,p-Dichlorodiphenyltrichloroethane (DDT); (6) Phenol; (7) Toluene; (8) 4-Chlorobiphenyl (PCB); (9) 3-Methylquinoline; (10) Benzonitrile; (11) n-Nonadecane; (12) Cyanuric acid; (13) Confusarin; (14) Pseudoguaianolide; (15) Xanthone; (16) Stilbene; (17) Cumene; (18) Actinidine; (19) Indole-3-acetonitrile; (20) Farnesol.

advances, which are often so reliant on novel chemistry. Given that few of the endosymbionts have been successfully cultured outside the host, understanding the microbe's detoxifying and degradative capacity is currently limited to inferring enzymatic function through comparative genomics and proteomics.

Among the numerous microbe-insect relationships, the termite-microbe relationship is one of the most complex and well studied. The termite gut contains several distinct microenvironments in which carbon and nitrogen from ligninocellulosic plant material and humus are transformed and assimilated by microorganisms [36,37]. The extreme microenvironments within the termite enable efficient scavenging and rapid transformation of recalcitrant organics. The termite diet, in particular lignin, shares structural similarities to recalcitrant organic pollutants, hence this ecosystem should encourage the proliferation of broad-substrate enzymes also applicable to xenobiotic degradation. Rhodococcus erythropolis TA421, a Grampositive bacterium isolated from a dry-wood termite ecosystem [38], has been shown to easily degrade several highly persistent xenobiotics, such as polychlorinated biphenyls (PCBs; Fig. 1; 8) [39].

Role of secondary metabolites in xenobiotic remediation Many plant-derived chemicals, including those generated from root turnover, stimulate microorganisms to biodegrade xenobiotics [40-44]. In particular, salicylate (Fig. 2; 22), which induces systemic acquired resistance (SAR) in plants [45], has been linked to the microbial degradation of naphthalene, a polycyclic aromatic hydrocarbon $(\mathrm{PAH})$ $[46,47]$. Chen and Aitken [48] demonstrated the ability of salicylate to greatly enhance the rate of removal of other PAHs, such as fluoranthene, pyrene (Fig. 1; 2), benz[a] anthracene, chrysene (Fig. 1; 3), and benz [a]pyrene. Salicylate elevated expression of $b p h A$, which encodes biphenyl dioxygenase, in the PCB-degrader Pseudomonas sp. Cam-1, whereas several terpenes (i.e. camphor (Fig. 2, 29), (s)-(+)carvone, p-cymene, and pinene) inhibited $b p h A$ activity at similar concentrations (1mM) [49]. Recognizing the plethora of plant-derived PAH analogues (Fig. 1), it is no surprise that 


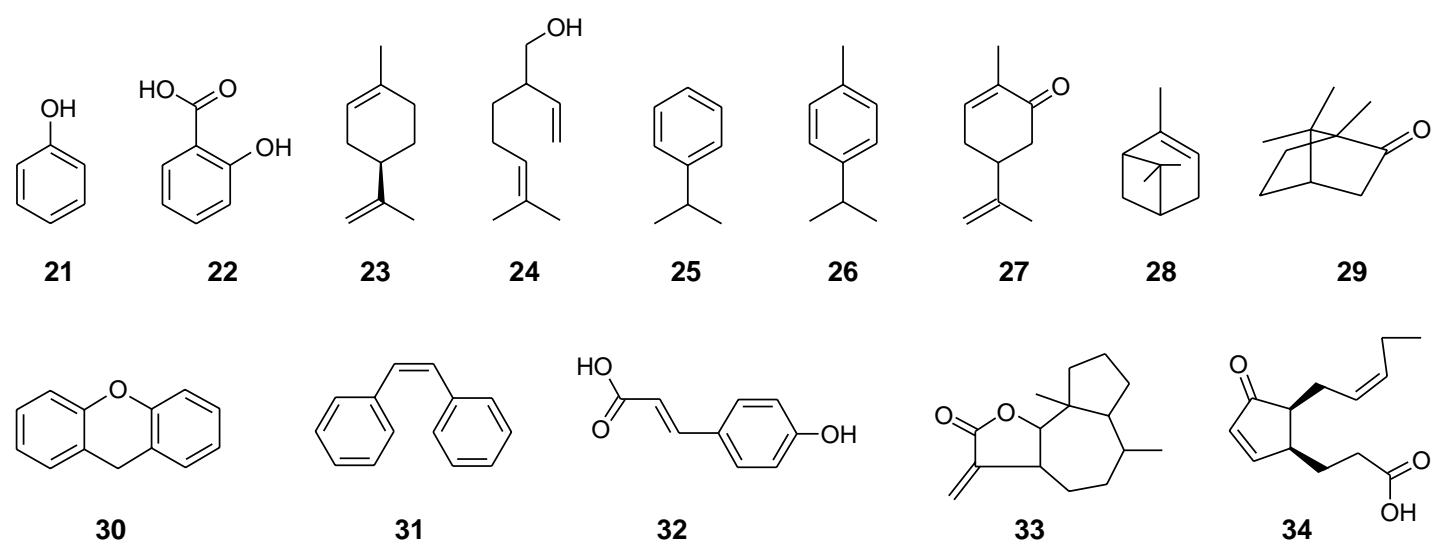<smiles>O=C1C[C@H](c2ccc(O)cc2)Oc2cc(O)cc(O)c21</smiles>

35<smiles>CN1CCC[C@H]1c1cccnc1</smiles>

39<smiles>O=c1c(O)c(-c2cc(O)c(O)c(O)c2)oc2cc(O)cc(O)c12</smiles>

36<smiles>COc1ccc(-c2c(O)c3c(OC)c4c(OC)c(C(C)C)oc4cc3oc2=O)cc1OC</smiles>

37<smiles>CC(C)=CCOc1c2c(cc3oc(=O)ccc13)OCC2</smiles>

38<smiles>COC(=O)C1CC2CCC(C1)N2C(=O)c1ccccc1</smiles>

40<smiles>CCCCCCCCCCCCCCCc1cccc(O)c1O</smiles>

41

Fig. 2. Structural diversity of common secondary plant metabolites (SPMEs). (21) Phenol; (22) Salicylic acid; (23) (R)-limonene; (24) Linalool; (25) Cumene; (26) Cymene; (27) Carvone; (28) $\alpha$-Pinene; (29) Camphor; (30) Xanthone; (31) Stilbene; (32) 4-Hydroxycinnamic acid; (33) Pseudoguaianolide; (34) Jasmonic acid; (35) Naringinen; (36) Myricetin; (37) $3^{\prime}, 4^{\prime}$-dihydrothonningine-C; (38) Isoimperatorin; (39) Nicotine; (40) Cocaine; (41) Urushiol (42) Farnesol.

the rhizosphere has been repeatedly shown to contain a diverse population of $\mathrm{PAH}$-degrading microorganisms [50]. Researchers have begun to investigate the efficacy of amending PAH-contaminated soil with salicylate in an effort to induce $\mathrm{PAH}$ degradation by indigenous soil microorganisms [51,52]. Presumably, the exogenous source of salicylate enhances the survival of $\mathrm{PAH}$-degrading microorganisms and induces the genes encoding enzymes involved in $\mathrm{PAH}$ degradation. Recent evidence also suggests a link between salicylate and PCB degradation [49,53,54].

Numerous challenges must be overcome to achieve successful in situ remediation of recalcitrant xenobiotics. When aerobically degraded, highly chlorinated xenobiotics such as PCBs provide the microorganism with neither carbon nor energy and thus they must be COMETABOLISED to be remediated. PCB-degrading microorganisms can be isolated easily from most soils by enrichment with biphenyl. Biphenyl is the COMETABOLITE ubiquitously chosen in laboratory research for the cometabolism of PCBs [55], but it cannot be applied to a PCB-contaminated site because it is harmful to the environment. Alternative cometabolites or inducers were therefore needed for in situ PCB bioremediation.

\section{Linking SPMEs to remediation}

Donnelly et al. [41] introduced the possibility that plantderived compounds, such as flavonoids, could support the growth of some PCB-degrading microorganisms and enhance PCB metabolism, thereby providing the first alternative to biphenyl. The best cometabolite and concentration was evaluated for each of the three PCB-degrading microorganisms, Ralstonia eutropha strain H850, Burkholderia cepacia LB400, and Corynebacterium sp. MB1. Each bacterium was subjected to a congener depletion assay [56], designed to show in a $24 \mathrm{~h}$ period the extent of congener degradation after growth on a particular flavonoid [41]. Naringin, a flavonoid found in grapefruit responsible for its bitter taste (Fig. $2 ; 35$ ), provided the best growth substrate for R. eutropha H850 and fostered the greatest metabolic activity towards PCB, of the 13 compounds tested. Myricetin, a flavonoid found in berries, herbs and pollen (Fig. 2; 36), induced the greatest PCB degradation in B. cepacia LB400, degrading 16 of the 19 congeners tested. Coumarin induced degradation of 13 congeners by Corynebacterium sp. MB1, exceeding the degradation achieved by biphenyl-grown controls. The authors suggested that a finely distributed network of plant roots could serve as a natural injection system, 
capable of dispensing cometabolites into the rhizosphere and inducing PCB degradation in indigenous microorganisms over long periods.

Focht [57] proposed that plant terpenes might be the natural substrate for PCB oxidation, rather than biphenyl. Hernandez et al. [58] subsequently demonstrated that soils enriched with orange peel (Fig. 2; 23), ivy leaves (Fig. 2; 41), pine needles (Fig. 2; 28) or eucalyptus leaves resulted in $10^{5}$ times more biphenyl utilizers $\left(10^{8} \mathrm{~g}^{-1}\right)$ than unamended soils $\left(10^{3} \mathrm{~g}^{-1}\right)$ simultaneously inducing AROCLOR 1242 degradation [58]. A similar study investigated the effect of pine needles and orange peel on the dissipation of Aroclor 1248 in rhizospheres of various plant species [59]. Similar PCB losses were observed in orange peel (54-59\% loss) and pine needle (44-55\% loss) treatments, including all planted and unplanted treatments.

Gilbert and Crowley [60] screened several terpenoid compounds, including $R$-carvone, $S$-carvone, cumene, carvacrol, thymol, $R$-limonene, $S$-limonene, $p$-cymene, and trans-cinnamic acid, for their ability to induce PCB biodegradation in Arthrobacter sp. strain B1B (Fig. 2; $\mathbf{2 3 , 2 5 , 2 6 , 2 7 , 3 2}$ ). S-Carvone, the principal chemical component of spearmint (Mentha spicata), induced the biotransformation of $62 \%$ of Aroclor 1242, or 26 of 32 identified peaks. Many of the SPMEs investigated are commonly found in dill and caraway seed, spearmint, pine needles, citrus, juniper, oregano, thyme and other aromatic plants $[58,60]$. The authors did not monitor degradation of each of the terpenoid compounds so it is difficult to assess if the induction was cometabolic, growth linked or otherwise. A subsequent study by the same authors investigated the efficacy of repeated applications of carvone-induced Arthrobacter sp. strain B1B onto Aroclor-1242-contaminated soils [61]. In two follow-up studies, Ralstonia eutropha H850 was repeatedly added to Aroclor-1242-contaminated soil as a member of a coinoculum with Arthrobacter sp. strain B1B [53] or Rhodococcus sp. ACS (the later study contained earthworms as a treatment variable) [54]. In both studies, Ralstonia eutropha H850 was grown on salicylic acid as the sole carbon source as well as the sole inducing substrate, whereas the other isolate was induced by carvone. A surfactant was added to improve bioavailability, enabling removal of $>60 \%$ of PCBs from the contaminated soils [53,54].

Tandlich et al. [62] used carvone and limonene to stimulate biodegradation of Delor 103 (another commercial mixture of PCBs) by Pseudomonas stutzeri. Additional PCB congener removal occurred after supplementing with terpenes compared with control-grown cultures [62]. Park et al. [63] demonstrated expression of the $b p h \mathrm{C}$ gene (2,3-dihydroxybipheyl 1,2-dioxygenase) in the PCB degrader Ralstonia eutropha H850, after induction by $(R)-(-)$-carvone (50 ppm). They concluded that carvone might induce a different degradative pathway, potentially generating different congener specificity to that of biphenylinduced cells.

Through the use of stereospecific degradation, Singer et al. [64] provided strong evidence for alternative PCBdegradative pathway(s) in bacteria, owing to changes in congener specificity after induction by two terpenes in the growth medium. The authors tested five PCB-degrading bacteria for their ability to differentiate between the ENANTIOMERS of four ATROPISOMERIC PCB congeners after growth in the presence of biphenyl, carvone or cymene. ENANTIOSELECTIVITY varied with respect to strain, congener and co-substrate. The authors concluded that the cosubstrate might induce an isoenzyme (similarly functioning enzyme), shifting the enantioselectivity because of the unique chirality of the isoenzyme's active site. Evidence of multiple degradative enzymes within a population has also been demonstrated for naphthalene. Researchers identified a naphthalene-degrading bacterium, Rhodococcus opacus M213, that is incapable of growth on salicylate, implying that there must be another pathway for this compound [65]. Later it was found that of 12 different PAH degraders only three could use both salicylate and phthalate (the two known intermediates of PAH degradation) as growth substrates and three were unable to use either substrate. The authors concluded that there is considerable heterogeneity among bacteria with respect to induction of PAH degradation [66].

This review has focused its examples largely on PCB degradation, but researchers have also found that terpenes can induce the degradation of other xenobiotic compounds (often through novel pathways), such as toluene, phenol (Fig. 1; 6,7) and trichloroethene (TCE) $[67,68]$. The diversity of substrates that are degraded in the presence of SPMEs might be easily explained when the SPME shares similar skeletal features with the pollutant (Fig. 1). Chemicals of similar form are more likely to fit within the active site of the same enzyme, whereas dissimilar structures will only share the same active site if it is a broad-substrate enzyme. The observed biotransformation of a pollutant in the presence of SPMEs with little structural similarity to the pollutant might not necessitate the activity of the classical pollutant-degrading enzyme; yet another enzyme, inducible by the SPME, might be responsible for transforming the pollutant [64]. One avenue of investigation is evaluating the induction of broad-specificity enzymes such as cytochrome P450.

Cytochrome P450 mono-oxygenases (hydroxylases), often referred to simply as CPY enzymes, catalyze oxygen insertion into many different kinds of substrates, including natural steroids, fatty acids and xenobiotics. They serve as the enzymes for drug metabolism, toxification and detoxication in the environment [69-71]. CPY enzymes are commonly found in animals, plants, insects and microorganisms, of which CPY101, cytochrome $\mathrm{P} 450_{\text {cam }}$, is amongst the best studied. Cytochrome $\mathrm{P} 450_{\text {cam }}$ (a bacterial camphor hydroxylase) catalyzes the stereoselective and regioselective hydroxylation of camphor (Fig. 2; 29) to 5-exo hydroxy camphor and is induced by a range of other SPMEs (Fig. 2; 23-28). Considerable resources in the public and private sector are being dedicated towards CYP research because of their importance in drug development, pollutant degradation and metabolism [70]. Jones et al. [72] manipulated the amino acid sequence of the cytochrome $\mathrm{P} 450_{\text {cam }}$ enzyme to try to increase the substrate range of the naturally occurring protein, with potential 
applications in bioremediation, biocatalysis and biosensors. The P450 enzyme could potentially be induced by a non-toxic SPME for the biotransformation of compounds of interest (e.g. pollutants and pharmaceuticals).

In plants, cytochrome $\mathrm{P} 450 \mathrm{~s}$ are involved in the biosynthesis of secondary products, including flavonoids and alkaloids (Fig. 2; 39,40), and hormones but also in the detoxification of herbicides and other xenobiotics. As previously discussed, plants also produce a range of SPMEs, many of which can be positively correlated with an increase in stress. Thus, the P450 enzymes within a plant might be linked to stimulating xenobiotic degradation in the rhizosphere through the production of SPMEs and directly through detoxification of the pollutant. Recent research has already demonstrated the potential to produce plants with the ability to overproduce secondary metabolites [73]. Hence, future studies might examine the potential for genetically modified plants to overproduce terpenes in contaminated soils as a response to the toxic stress, in turn stimulating biotransformation of the pollutant by rhizosphere microbial populations.

\section{Future perspective}

Although there is considerable evidence that many secondary plant metabolites can stimulate microbial degradation of xenobiotics and broaden the spectrum of their activity, we are still ignorant of the regulatory mechanism within microorganisms. What is more certain is that the use of plant substrates for stimulating desired microbial reactions holds great promise for biotechnological exploitation, both in terms of bioremediation of the environment and biotransformation of chemicals in reactor-based systems. To have at our disposal a range of INDUCERS that, when introduced into the environment, stimulates (rate and extent) microbial xenobiotic degradation would have enormous advantages. First, SPMEs are often effective at very low concentrations and inherent in their natural origins is that they are 'environmentally friendly'. There would be far fewer concerns about introducing them into sensitive sites such as aquifers that supply drinkable water. Given that they are effective at or below their solubility in water, they can be easily applied to poorly accessible sites in the sub-soil or deep aquifers. With an increased understanding it should be possible to identify more precisely which inducers or classes of compounds are most effective at stimulating microbial degradation of specific contaminants or classes of chemicals. With further study it would be convenient to identify inducers that are effective against a broad range of contaminants or to construct cocktails that are tailormade for mixed contaminated sites. The use of SPMEs to stimulate microbial degradation of pollutants offers the opportunity to develop sustainable systems for sites that are particularly vulnerable to regular contamination events. By introducing plants to the site that naturally produce appropriate secondary metabolites, a readily available and cheap barrier could be established for long-term site protection. With time, the chemical manufacture of pesticides might provide an inducer that complements the primary product as an 'antidote' towards its remediation. One could indulge in a scenario in which a crop requires the use of a pesticide or herbicide for only a finite period of time, after which point the pesticide becomes unnecessary and environmentally hazardous. It might be possible to administer a single application of one or several SPMEs, after which the pesticide is substantially biodegraded.

In parallel to the role of SPMEs in bioremediation, the potential for SPMEs to stimulate microbial activity and extend the spectrum of their catabolic ability holds great promise for industrial processes whereby microorganisms are exploited to undertake specific chemical transformations resulting in valued products. Not least, selection of appropriate plant metabolites could increase rates of reactions and yields, thus making the process more economical. The recent observation [71] that the addition of appropriate plant products can favor the formation of a specific enantiomer product represents a particular exciting opportunity. A major cost when dealing with the products of enantiomer reactions is the separation of the chiral mixtures. Such costs would be reduced if the initial microbial transformation could be directed by using appropriate SPMEs to favor formation of the chiral product of value.

The use of SPMEs for enhancing the ability of microorganisms to remediate the environment and produce high value products by biotransformation is a promising technology that is very much in its infancy. To realize the potential of this approach it will be essential to improve our ability to determine the range of plant products that can help microorganisms express and extend their catabolic potential and, importantly, determine the mechanism by which this stimulation occurs. New tools and information made available through genomic and proteomic arrays will help to meet these challenges but effective multidisciplinary research is also vital. The results of thousands of years of plant-microbe-insect interactions have provided us with a real opportunity to harness and direct the phenomenal catabolic ability of microorganisms. It is up to us, however, to first recognize this potential and second understand how to exploit it effectively in biotechnological applications.

\section{References}

1 Burdon, J. (2001) Are the traditional concepts of the structures of humic substances realistic. Soil Sci. 166, 752-769

2 Marschner, H. (1995) Mineral nutrition of higher plants, Academic Press

3 Salt, D.E. et al. (1998) Phytoremediation. Annu. Rev. Plant Physiol. Plant Mol. Biol. 49, 643-668

4 Jones, D. (1998) Organic acids in the rhizosphere - a critical review. Plant Soil 205, 24-44

5 Knee, E.M. et al. (2001) Root mucilage from pea and its utilization by rhizosphere bacteria as a sole carbon source. Mol. Plant Microbe Interact. $14,775-784$

6 Gerhardson, B. (2002) Biological substitutes for pesticides. Trends Biotechnol. 20, 338-343

7 Hadacek, F. (2002) Secondary metabolites as plant traits: current assessment and future perspectives. CRC Crit. Rev. Plant Sci. 21, $273-322$

8 Dixon, R. (2001) Natural products and plant disease resistance. Nature 411, 843-847

9 Paul, N.D. et al. (2000) Coping with multiple enemies: an 
integration of molecular and ecological perspectives. Trends Plant Sci. $4,220-225$

10 Gershenzon, J. and Croteau, R. (1991) Terpenoids. In Herbivores: Their Interactions with Secondary Plant Metabolites: The Chemical Participants (Vol. 1) (Rosenthal, G.A. and Berenbaum, M.R., eds) pp. 165-220, Academic Press

11 Harborne, J.B. (1991) Monoterpenes. In Methods in Plant Biochemistry: Terpenoids (Vol. 7) (Charlwood, B.V. and Banthorpe, D.V., eds) pp. 1-28, Academic Press

12 Iscan, G. et al. (2002) Antimicrobial screening of Mentha piperita essential oils. J. Agric. Food Chem. 50, 3943-3946

13 Charlwood, B.V. and Charlwood, K.A. (1991) Monoterpenes. In Methods in Plant Biochemistry (Vol. 7-Terpenoids) (Charlwood, B.V. and Banthorpe, D.V., eds) pp. 43-98, Academic Press

14 Harborne, J.B. (1989) General procedures and measurement of total phenolics. In Methods in Plant Biochemistry (Vol. 1: Plant Phenolics) (Harborne, J.B., ed.), pp. 1-28, Academic Press

15 Berenbaum, M.R. (1991) Coumarins. In Herbivores: Their interactions with secondary plant metabolites: The Chemical Participants (Vol. 1) (Rosenthal, G.A. and Berenbaum, M.R., eds) pp. 221-296, Academic Press

16 Rausher, M.D. (2001) Co-evolution and plant resistance to natural enemies. Nature 411, 857-864

17 Bouarab, K. et al. (2002) A saponin-detoxifying enzyme mediates suppression of plant defenses. Nature 418, 889-892

18 Farmer, E.E. (2001) Surface-to-air signals. Nature 411, 854-856

19 Kessler, A. and Baldwin, I.T. (2002) Plant responses to insect herbivory: the emerging molecular analysis. Annu. Rev. Plant Biol. $53,299-328$

20 Valladares, G.R. et al. (2002) Phytochemical induction by herbivores could affect quality of essential oils from aromatic plants. J. Agric. Food Chem. 50, 4059-4061

21 Arimura, G-I. et al. (2000) Herbivory-induced volatiles elicit defense genes in lima bean leaves. Nature 406, 512-515

22 Pare, P.W. and Tumlinson, J.H. (1997) De novo biosynthesis of volatiles induced by insect herbivory in cotton plants. Plant Physiol. 114, $1161-1167$

23 DeMoraes, C.M. et al. (1998) Herbivore-infested plants selectively attract parasitoids. Nature $393,570-573$

24 Dicke, M. (1994) Local and systemic production of volatile herbivoreinduced terpenoids: their role in plant-carnivore mutualism. J. Plant Physiol. 143, 465-472

25 Meijden, E.V.D. and Klinkhamer, P.G.L. (2000) Conflicting interests of plants and the natural enemies of herbivores. Oikos 89, 202-208

26 Pare, P.W. and Tumlinson, J.H. (1999) Plant volatiles as a defense against insect herbivores. Plant Physiol. 121, 325-331

27 Strycharz, S. and Shetty, K. (2002) Effect of Agrobacterium rhizogenes on phenolic content of Mentha pulegium elite clonal line for phytoremediation applications. Process Biochem. 38, 287-293

28 Singh, U.P. et al. (2002) Plant growth-promoting rhizobacteriamediated induction of phenolics in pea (Pisum sativum) after infection with Erysiphe pisi. Curr. Microbiol. 44, 396-400

29 Sylvia, D.M. and Williams, S.E. (1992) Vesicular-arbuscular mycorrhizae and environmental stress Mycorrhizae in Sustainable Agriculture, ASA, CSSA, SSSA, pp. 101-124

30 Saikkonen, K. et al. (1998) Fungal endophytes: a continuum of interactions with host plants. Annu. Rev. Ecol. Syst. 29, 319-343

31 Hammon, K.E. and Faeth, S.H. (1992) Ecology of plant-herbivore communities: a fungal component? Nat. Toxins 1, 197-208

32 Baldwin, I.T. et al. (2001) Merging molecular and ecological approaches in plant-insect interactions. Curr. Opin. Plant Biol. 4, $351-358$

33 Grodnitzky, J.A. and Coats, J.R. (2002) QSAR evaluation of monoterpenoids' insecticidal activity. J. Agric. Food Chem. 50, $4576-4580$

34 Dohlen, C.D.V. et al. (2001) Mealybug $\beta$-proteobacterial endosymbionts contain $\gamma$-proteobacterial symbionts. Nature $412,433-436$

35 Wilkinson, T.L. et al. (2001) The impact of host plant on the abundance and function of symbiotic bacteria in an aphid. J. Exp. Biol. 204, 3027-3038

36 Lilburn, T.G. et al. (1999) Phylogenetic diversity of termite gut Spirochaetes. Environ. Microbiol. 1, 331-345
37 Brune, A. and Friedrich, M. (2000) Microecology of the termite gut: structure and function on a microscale. Curr. Opin. Microbiol. 3, 263-269

38 Chung, S-Y. et al. (1994) Isolation and characterization of a grampositive polychlorinated biphenyl-degrading bacterium, Rhodococcus erythropolis strain TA421, from a termite ecosystem. Biosci. Biotechnol. Biochem. 58, 2111-2113

39 Maeda, M. et al. (1995) Multiple genes encoding 2,3-dihydoroxybiphenyl 1,2-dioxygenase in the gram-positive polychlorinated biphenyldegrading bacterium Rhodococcus erythropolis TA421, isolated from a termite ecosystem. Appl. Environ. Microbiol. 61, 549-555

40 Miya, R.K. and Firestone, M.K. (2001) Enhanced phenanthrene biodegradation in soil by slender oat root exudates and root debris. J. Environ. Qual. 30, 1911-1918

41 Donnelly, P.K. et al. (1994) Growth of PCB-degrading bacteria on compounds from photosynthetic plant. Chemosphere 28, 981-988

42 Haby, P.A. and Crowley, D.E. (1996) Biodegradation of 3-chlorobenzoate as affected by rhizodeposition and selected carbon substrates. J. Environ. Qual. 25, 304-310

43 Fletcher, J.S. and Hegde, R.S. (1995) Release of phenols by perennial plant roots and their potential importance in bioremediation. Chemosphere 31, 3009-3016

44 Isidorov, V. and Jdanova, M. (2002) Volatile organic compounds from leaves litter. Chemosphere 48, 975-979

45 Meyer, G.D. et al. (1999) Nanogram amounts of salicylic acid produced by the rhizobacterium Pseudomonas aeruginosa 7NSK2 activate the systemic acquired resistance pathway in bean. Mol. Plant Microbe Interact. 12, 450-458

46 Yen, K-M. and Gunsalus, I.C. (1982) Plasmid gene organization naphthalene/salicylate oxidation. Proc. Natl. Acad. Sci. U. S. A. 79, 874-878

47 Meer, J.R.V.D. et al. (1992) Molecular mechanisms of genetic adaptation to xenobiotic compounds. Microbiol. Rev. 56, 677-694

48 Chen, S-H. and Aitken, M.D. (1999) Salicylate stimulates the degradation of high-molecular weight polycyclic aromatic hydrocarbons by Pseudomonas saccharophilia P15. Environ. Sci. Technol. $33,435-439$

49 Master, E.R. and Mohn, W.W. (2001) Induction of $b p h A$, encoding biphenyl dioxygenase, in two polychlorinated biphenyl-degrading bacteria, psychrotolerant Pseudomonasstrain Cam-1 and mesophilic Burkholderia strain LB400. Appl. Environ. Microbiol. 67, 2669-2676

50 Daane, L.L. et al. (2001) Isolation and characterization of polycyclic aromatic hydrocarbon-degrading bacteria associated with the rhizosphere of salt marsh plants. Appl. Environ. Microbiol. 67, 2683-2691

51 Ogunseitan, O.A. et al. (1991) Effect of 2-hydroxybenzoate on the maintenance of naphthalene-degrading pseudomonas in seeded and unseeded soil. Appl. Environ. Microbiol. 57, 2873-2879

52 Colbert, S.F. et al. (1993) Use of an exotic carbon source to selectively increase metabolic activity and growth of Pseudomonas putida in soil. Appl. Environ. Microbiol. 59, 2056-2063

53 Singer, A.C. et al. (2000) Bioremediation of polychlorinated biphenylcontaminated soil using carvone and surfactant-grown bacteria. Appl. Microbiol. Biotechnol. 54, 838-843

54 Singer, A.C. et al. (2001) Contribution of earthworms to PCB bioremediation. Soil Biol. Biochem. 33, 765-776

55 Brunner, W. et al. (1985) Enhanced biodegradation of polychlorinated biphenyls in soil by analogue enrichment and bacterial inoculation. J. Environ. Qual. 14, 324-328

56 Bedard, D.L. et al. (1986) Rapid assay for screening and characterizing microorganisms for the ability to degrade polychlorinated biphenyls. Appl. Environ. Microbiol. 51, 761-768

57 Focht, D.D. (1995) Strategies for the improvement of aerobic metabolism of polychlorinated biphenyls. Curr. Opin. Biotechnol. 6, 341-346

58 Hernandez, B.S. et al. (1997) Terpene-utilizing isolates and their relevance to enhanced biotransformation of polychlorinated biphenyls in soil. Biodegradation 8, 153-158

59 Dzantor, E.K. and Woolston, J.E. (2001) Enhancing dissipation of Aroclor 1248 (PCB) using substrate amendment in rhizosphere soil. J. Environ. Sci. Health 36, 1861-1871

60 Gilbert, E.S. and Crowley, D.E. (1997) Plant compounds that induce 
polychlorinated biphenyl biodegradation by Arthrobacter sp. strain B1B. Appl. Environ. Microbiol. 63, 1933-1938

61 Gilbert, E.S. and Crowley, D.E. (1998) Repeated application of carvoneinduced bacteria to enhance biodegradation of polychlorinated biphenyls in soil. Appl. Microbiol. Biotechnol. 50, 489-494

62 Tandlich, R. et al. (2001) The effect of terpenes on the biodegradation of polychlorinated biphenyls by Pseudomonas stutzeri. Chemosphere 44 , 1547-1555

63 Park, Y-I. et al. (1999) Induction by carvone of the polychlorinated biphenyl (PCB)-degradative pathway in Alcaligenes eutropha H850 and its molecular monitoring. J. Microbiol. Biotechnol. 9, 804-810

64 Singer, A.C. et al. (2002) Differential enantioselective transformation of atropisomeric polychlorinated biphenyls by multiple bacterial strains with differing inducing compounds. Appl. Environ. Microbiol. $68,5756-5759$

$65 \mathrm{Uz}$, I. et al. (2000) Characterization of the naphthalene-degrading bacterium, Rhodococcus opacus M213. FEMS Microbiol. Lett. 185, 231-238

66 Bogan, B.W. et al. (2001) Limited roles of salicylate and phthalate in bacterial PAH bioremediation. Bioremed. J. 5, 93-100
67 Kim, D. et al. (2002) Three separate pathways for the initial oxidation of limonene, biphenyl, and phenol by Rhodococcus sp. strain T104. J. Microbiol. 40, 86-89

68 Davrock, B. et al. (1992) Isopropylbenzene (cumene) - a new substrate for the isolation of trichloroethene-degrading bacteria. Arch. Microbiol. 158, 9-13

69 Scott, J.G. and Wen, Z. (2001) Cytochromes P450 of insects: the tip of the iceburg. Pest Manag. Sci. 57, 958-967

70 Guengerich, F.P. (2001) Common and uncommon cytochrome P450 reactions related to metabolism and chemical toxicity. Chem. Res. Toxicol. 14, 611-650

71 Noordermeer, M.A. et al. (2001) Fatty acid hydroperoxide lyase: a plant cytochrome $\mathrm{P} 450$ enzyme involved in wound healing and pest resistance. Chem. Biochem 2, 494-504

72 Jones, J.P. et al. (2001) Oxidation of polychlorinated benzenes by genetically engineered CYP101 (cytochrome P450cam). Eur. J. Biochem. 268, 1460-1467

73 Verpoorte, R. and Memelink, J. (2002) Engineering secondary metabolite production in plants. Curr. Opin. Biotechnol. 13, 181-187

\section{Do you want to reproduce material from a Trends journal?}

This publication and the individual contributions within it are protected by the copyright of Elsevier Science.

Except as outlined in the terms and conditions (see p. ii), no part of any Trends journal can be reproduced, either in print or electronic form, without written permission from Elsevier Science.

Please address any permission requests to:

Rights and Permissions,

Elsevier Science Ltd,

PO Box 800, Oxford, UK OX5 1DX. 